\title{
Discussion on the Operation Management Measures of Clothing Factory -- Set Ri Mao Clothing Factory as an Example
}

\author{
Jihong Zhang \\ College of Clothing Management, Jiangxi Institute of Fashion Technology, Nanchang Jiangxi, \\ 330201, China
}

\begin{abstract}
Key words: Ri Mao Clothing Factory, Construction Environment, Production System, Design, Operation Management, Labor Productivity.
\end{abstract}

\begin{abstract}
In order to improve product quality, lower production cost, ensure long-term sustainable survival and development, enterprises have to meet the market development needs and customers' requirements, solve problems facing in the production process with reasonable operation management methods to maintain the production rate and economic benefit at a high level. This paper set Ri Mao Clothing Factory as an example, discussed its factory construction background and business environment. This paper set the design requirements, principle and operating environment of clothing production system as foreshadowing to discuss its relevant operation management measures, hoping to improve the labor productivity of Ri Mao Clothing Factory.
\end{abstract}

\section{Introduction}

Ri Mao Clothing Factory decided its construction standard based on comprehensive analysis on its internal and external environment, its adopted JIT thoughts for its production system design, tried to design products which can meet customer needs, and to realize the production characteristics of "multi-type, small batch and rapidness" and to optimize the enterprise cost. This is also the core content of Ri Mao Clothing Factory’s operation management.

\section{Construction background of Ri Mao Clothing Factory}

\section{Construction background}

Ri Mao Clothing Factory is a limited company, its main business is import and export of knitted dress, mainly exporting a large number of apparel supplies to Japan. Since being founded, its annual foreign exchange earnings is more than 4 million dollar, the specific business process of its export business is first to get product order from Japanese customer, then to purchase finished products from the specified partner in China, at last to entrust the agent company to do the export business of dress-goods. $80 \% \sim 90 \%$ of products in the company need commodity inspection, it only can be exported to Japan on the basis of meeting quality standard. Therefore, Ri Mao Clothing Factory has proposed six aspects of construction requirements, hoping to solve the present problems.

First of all, Ri Mao existed as a cooperative party with other factories before constructing its own factory, thus its main problem was that employees' overall quality was low, and its management had deficiencies, which constantly increased its product repair rate, according to figures, its product repair rate reached up to $20 \% \sim 50 \%$, many products need to be repaired for the bad management, the main problem was serious quality problem and late delivery, which caused frequent claim phenomenon, which caused huge loss to the factory. Therefore, it is necessary to construct Ri Mao Clothing Factory with scientific management to solve management operation problems of the former factory.

Secondly, corporate customers were relatively concentrated, and with the change of market demand, order requirements from Japan also tend to be "varieties and multiple". Because the factory cannot realize quantity production, it was afraid to accept big orders. Besides, the technological 
requirements of order products were complicated, which may be unable to realize smooth delivery, thus, in order to optimize technology, improve labor productivity and meet customer demands, it is necessary to build Ri Mao Clothing Factory.

Thirdly, the quality of the knitted fabrics affected the quality of the finished products, main problems are printing defect, dyeing and finishing defect and so on. In order to solve above problems, ought to take supplementary material as main measure, require fabric supplier to supplement materials timely, in consideration of that the purchase of some fabrics is not convenient and its delivery time is also hard to be guaranteed, thus needs to build Ri Mao Clothing Factory. Integrate the fabric and tailor two links into an independent link, mutual restraints and make up in terms of production technology, at the same time set up a fabric purchase government to reduce purchase cost for Ri Mao Clothing Factory.

Fourthly, considering the problem of short production cycle and combining the present actual condition of the factory, in order to avoid delay delivery time and suffer from credit crisis, it is necessary to build Ri Mao Clothing Factory to make arrangement of delivery time and production by themselves to realize reasonable production and timely delivery thus to realize the stable operation management system and create more export profit for the factory.

Fifthly, the appointed export port and inspection center of Ri Mao Clothing Factory are set in Da Lian, but most of its cooperative factories are located in other places of Li Ning Province, such as An Shan, Zhao Yang, Ying Kou, etc. Thus it is necessary to build Ri Mao Clothing Factory in local Da Lian, one for convenient transportation, the other for saving logistics cost.

Sixthly, from the long-term development of the enterprise, it is also need to build a base with independent production system to meet the actual needs that caused by the increase of fixed assets of clothing factory to make the enterprise more competitive, which is also conform to the object of long-term sustainable development.

\section{Domestic clothing industry environment}

In order to meet the requirements of domestic clothing industry environment and follow up the pace, and considered the circumstances change to build Ri Mao Clothing Factory. This is also to adapt to the steadily rising demand change of the domestic market, according to China's customs statics, the export volume of dress-goods to Japan reached 90.24 billion dollars, increased by $13.2 \%$ for the same period in 2014. Though the raw material price decreased slowly as a whole, the present product price continuously rises. And the production and selling of products are gradually to be synchronized, which make the export economic profit be improved. Among which, the clothing export industry is also the most profiting light industry in our country, the profit amount is more that 9 billion dollars.

\section{The basic condition for the construction of Ri Mao Clothing Factory}

In order to construct a better factory operation management system, Ri Mao Clothing Factory also gradually transits the market from sellers' market to buyers' market, to meet the demand of modern clothing production and the actual requirements of multispecies, short amount and short production cycle. Besides, Ri Mao Clothing Factory also should conform to the requirements of present market economic changes, try to improve its market competitiveness, put forward a service system with high quality, low cost and short production cycle, to adapt to the elastic demand of current market, and to construct a comprehensive production system that can react flexibly for the dress-goods.

Actually, before Ri Mao Clothing Factory being established, what the enterprise chased is the blind production mode that with few species and in big quantity, but since Ri Mao Clothing Factory is established, it began to apply the non-stock sales operation mode, realize true production line "fast production and fast selling”. The following is the production system reasonably process of Ri Mao Clothing Factory.

The factory established a production system that suitable for the export sales speed (target: shorten the production cycle, improve adaptability to changes in the market) $\rightarrow$ establish small batch 
production mechanism (target:realize homogenized organized production mode and shorten change time) $\rightarrow$ shorten production cycle $\rightarrow$ simplify production mode (target: build production line with small batch but multispecies).

As above mentioned process, Ri Mao Clothing Factory can realize homogenized organized production mode on the management of production mechanism operation, realize the same pace of sales and production, construct more flexible JIT production system, thus to meet its development demand $^{[1]}$.

\section{Analysis on the production system design of Ri Mao Clothing Factory}

\section{Requirements on the basic production system design}

The production system of Ri Mao Clothing Factory requires to undertake the basic thought of Just In Time, the key content of this thought is production on time, its purpose is to keep the minimum inventory from raw material to finished product and the maximum batch production ratio. If it can realize JIT target, it can realize cost saving from all aspects of the factory's production activities. For example, when Ri Mao Clothing Factory sells a product, its internal market system terminal will pull a product thus to form the order requirement for production line, that is to say, its supplementary products will be directly supplemented in the upstream process, which forms the processing of new products, finally finish product production, then wait for output. From the aspect of solving problems, JIT realized the following 3 contents: solve the time, production action, stock and wastes problems, solved the bottleneck problem on some production technology, realized assembly line production mode. Besides, JIT put forward brand new production requirements, that is all staff participation, continuous improvement, total quality management and small scale production. Meeting above requirements, the operation management measures of Ri Mao Clothing Factory can be officially launched.

\section{Principle of the basic production system design}

Ri Mao Clothing factory mainly follows three points on the principle of production system design: firstly, have a balanced production plan. Make a full understanding of the production species of the clothing factory, control the production time, and try to be comprehensively balanced. This principle not just require the balance on quantity, and also not just require the balance of traditional production mode, but the balanced production mode, ensure the equal proportion of working procedure, batch, construction period, sales cycle.

Secondly, apply "three timely" principle according to JIT requirements, produce the output and production time of the standardized product. Therefore, Ri Mao Clothing Factory should officially sign production output contract with customers, accomplish production process according to the agreed delivery time and guarantee the quality ${ }^{[2]}$.

\section{Analysis on the operation management measures of Ri Mao Clothing Factory}

Ri Mao Clothing Factory built a operation management system for its own long-term stable production, among which including positing management, labor management, quality management and equipment management. The following will analyze one by one.

\section{Analysis on positing management}

Positing management is the 5S management activities pushed out by Ri Mao Clothing Factory after its establishment, it includes clearing up, reorganizing, cleaning, cleanness and attainment, five aspects. Firstly, through clearing up to wipe out things that have nothing to do with the production line, then reorganize to the required things for the production site and put them on the right position for production. The key point of positing management is requiring human and things to form organic 
combination, avoid the appearance of labor in vain and unsafe factors, and determine the 3 states between human and things on the production line according to the real production site.

State 1: when the human and things are combined tightly, it needs to realize that production staff can get things easily, thus to improve production efficiency. For example, in the clothing production, sewing machine craftsman should ensure that hot iron, tool scissors should be by hand, thus to ensure he can realize tailor and ironing.

State 2: when human combines with things but need to find, for example, some finished clothing that did not get stock management or finished clothing that cannot be repaired in a short time.

State 3: when human and things do not need to be combined. That is some debris or wastes that interfere normal production, such as scraps, cuttings and so on.

Generally speaking, the target of the positing management of Ri Mao Clothing Factory is to store the things in state 1 , change things in state 2 and treat things in state 3.

\section{Analysis on labor management}

Labor management mainly includes specification on labor system and effective evaluation on wages. Set salary evaluation as an example, the basic principle it needs to follow is to reflect efficiency privilege and equity principle. In Ri Mao Clothing Factory, it mainly apply the principle of more pay for more work, this also can reasonably pull the gap between labors, to elect excellent employees. While the piece wage objectively and exactly reflect the labors' real amount of labor, it effectively combined labor reward with fruits of labor. Set the working procedure of rolling up bottom margin, first to conform the wage level of each procedure of this project, then according to the standard working time (12s) to calculate the piece wage (set 8 hours working time per day as an example),thus the daily output and monthly output of rolling up bottom margin is:

Daily output $=3600 \times 8 / 12=2400$ (pieces)

Monthly output $=30 \times 2400=72000$ (pieces)

Calculate labors' real wages according to raw material price, management cost, product profit including the average urban wage.

\section{Analysis on quality management}

Quality management is an essential link of modern enterprises' development competitiveness, in order to realize timely and high quality delivery, chase for no defect products, Ri Mao Clothing Factory comprehensively applied total quality control mode, this mode effectively mobilized the function of quality supervision branches, it was set as the necessary element for Ri Mao's effective operation. Meanwhile, TQC also takes quality as its core, takes profit, production, time as the overall operation activity characteristics of the target, hoping to infiltrate this operation management concept into every employees' heart, then matched with the concept of "producing no defect product", combine 5S management content on the basis of reducing cost to perfect management mechanism and strengthen the management cycle process among procedures.

\section{Analysis on equipment management}

In Ri Mao Clothing Factory, equipment operation management measures are mainly based on the production, quality and cost of clothing products to realize. Therefore, the factory should undertake the principle of "three well" of manage well, use well and maintain well, and maintain the machine equipment timely, thus to improve its function, then to adjust the technical state to most stable and to give full play of the highest performance of the equipment. Take the maintenance of sewing machine equipment, it requires when the equipment has general failure, it can recover function through simple repair, realize the regular checking and repair process of the equipment, this is also called as "preventive maintenance". While after the sewing machine equipment recovers to normal safety operation after debugging, it should get into the "productive maintenance" process ${ }^{[3]}$. 


\section{Conclusion}

The construction of management measures of Ri Mao Clothing Factory is to adapt to current highly competitive socialist market economy environment, on the premise of meet the technological development demand to improve operation management mechanism, establish comprehensive production mechanism match with the market environment. Meanwhile, make production plan according to demand and amount and time, to save cost and explore new way for the enterprise.

\section{References}

[1] Ke Mingli. Analysis and Practice of the Ri Mao Clothing Factory Design. Dalian University of Technology, 2001.35-44.

[2] Zhong Hong. Discussion and Analysis on Improving the Operation Management System of JY Clothing. Xiamen University, 2008.46-76.

[3] Li Tao, Liu Wenfang. Analysis on the Supply Chain Management Mode of Garment Foreign Trade Corporations.Commercial Economy Research, 2015(15):91-93. 